\title{
The effects of sensory stimulation in inducing or intensifying the "transport response" in white rats
}

\author{
CHRISTOPHER WILSON \\ Lafayette College, Easton, Pennsylvania
}

\begin{abstract}
A series of experiments was carried out to investigate the ability of different types of stimulation to induce or intensify the "transport response" in 15-day-old rat pups when an initial stimulus, a pinch to the nape of the neck, produced either no transport response or only a very weak response. Rat pups given supplemental tactile stimulation, in the form of either an airpuff to the ventrum or a pinch to the tail, showed consistently stronger response intensities than when given only a pinch to the nape of the neck. Visual and auditory stimuli produced no increment in response intensities over those with only a dorsal pinch. Two additional experiments were carried out. The first investigated the ability of a puff of mist from an atomizer to induce a transport response in rats too old to show a strong response. The second investigated the induction of the transport response with supplemental stimulation in 19-day-old pups given haloperidol. Results suggest a nondopaminergic system subserving the response facilitation seen with supplemental tactile stimulation.
\end{abstract}

The predominant behaviors at different times during an organism's lifetime tend to be beneficial to survival. In nonprecocial animals, an infant may show a specific response to an environmental stimulus that might be quite different from that shown by an adult to the same stimulus. The infant behaviors should not be viewed as miniature adult behaviors but as specific to the young organism (Spear \& Ristine, 1982) and to its relationship with its parents and/or its environment.

In nonprecocial animals, many specific behaviors shown by an infant must be coordinated with behaviors shown by the mother to insure the survival of the young. Brewster and Leon (1980) described such an interaction in rats that enables the mother to transport the infant from one location to another. This "transport response" (TR) is elicited by the mother's biting the infant's back or the nape of its neck. Rather than responding passively, like some species (Kleiman, 1972; Sauer, 1967), the infant rat actively responds by extending and adducting its forelimbs and flexing and adducting its hindlimbs. This produces a somewhat compact package for transport. The ecological value of the response was demonstrated by Brewster and Leon (1980), who lightly anesthetized infant rats just prior to maternal retrieval. This blocked the limb adduction in the infant, and, consequently, the mother had a more difficult time in transporting the infant, tending to drag it and stumble over it. That tactile stimulation is also important is

The author thanks B. H. Cohen and Howard F. Gallup of Lafayette College for extensive help in the preparation of this manuscript. The author also expresses appreciation to Jim Delabar for his kind help in developing the experimental situation and collecting data, and to Lisa S. Anderson, Stuart E. Greenberg, and Suzanne W. Halm for their help in collecting and scoring the data. The author's mailing address is: Department of Psychology, Lafayette College, Easton, PA 18042. indicated; a sensory block with Xylocaine to the stimulated area in the pups tended to block the limb adduction in the pups. Brewster and Leon (1980) further demonstrated that tactile stimulation to the back and to the nape of the neck is especially effective in eliciting an intense response, whereas tactile stimulation to other areas produces only a weak response. Brewster and Leon (1980) did not rule out the possibility that other stimuli might have an effect on the TR.

The possible mechanisms underlying the TR in rat pups have been previously reported. Haloperidol, a potent postsynaptic dopamine antagonist (Siegel, Albers, Agranoff, \& Katzman, 1981), produces a dose-dependent reduction in the TR in 19-day-old rat pups (Wilson, Cullen, \& Sendell, 1984). On the other hand, administration of apomorphine, a dopaminergic agonist that binds to dopamine receptors and mimics the effects of endogenous dopamine (Siegel et al., 1981), can reinstate an intense TR in rats normally too old to show a strong response (Wilson, 1985). Interestingly, dopamine systems have been reported to undergo development during the first 3 to 4 postpartum weeks (Loizou, 1969, 1972). These results seemed to indicate that the TR very well might be subserved by an endogenous dopamine system. During the course of the experiments involving the administration of haloperidol (Wilson et al., 1984) and apomorphine (Wilson, 1985), it was observed that when a pinch to the nape of the neck was less than adequate to produce a strong TR, a supplemental airpuff to the infant's ventrum seemed to sufficiently intensify the TR. The value of such a phenomenon might be to intensify the TR when the mother does not have an appropriate grasp or when the pup's sensory mechanisms for detecting specifically the dorsal pinch prove inadequate and the pup is left to drag on the 
ground. A third alternative might be that an increment in response intensity with additional stimulation is a compensatory response, brought into play if the normal neurochemical systems elicited by the mother's grasp are not well developed.

Experiments $1 \mathrm{a}, 1 \mathrm{~b}$, and $1 \mathrm{c}$ investigated the types of stimuli that might potentiate the transport response when a pinch to the nape of the neck proves an inadequate stimulus. Experiment 2 investigated the ability of supplemental stimuli to elicit a TR in 33-day-old rats when the normal mechanisms underlying the TR are suppressed. Experiment 3 investigated the possibility that the neurochemical mechanism underlying a potentiated response in 19-day-old animals is the same mechanism reported in previous research (Wilson, 1985; Wilson et al., 1984).

\section{GENERAL METHODS}

\section{Subjects}

Subjects in Experiments 1a, 1b, and 1c were 72 Sprague-Dawley albino rats, 15 days of age at the time of testing. Fifteen-day-old pups were used because they are mature enough to show a TR and because they are small enough to be suspended with the mechanical device we chose to use. Subjects in Experiment 2 were 32 Sprague-Dawley albino rats, 33 days of age at the time of testing. Subjects in Experiment 3 were 40 Sprague-Dawley albino rats. Since Experiment 3 was, in part, a replication of previous work (Wilson et al., 1984), subjects in this experiment were 19 days of age at the time of testing.

Litters were derived from breeding colonies maintained in the Department of Psychology at Lafayette College. All litters were housed with their mothers in clear Plexiglas cages, in a room kept at $22^{\circ} \mathrm{C}$, on a 12:12-h light:dark cycle with lights on at $0700 \mathrm{~h}$. Testing occurred between 0900 and $1200 \mathrm{~h}$.

\section{Materials}

The haloperidol used in Experiment 3 was supplied by McNeil Pharmaceuticals, Spring House, Pennsylvania.

\section{Procedures}

Approximately 1 week prior to parturition, pregnant female rats were placed in clear Plexiglas breeding chambers containing bedding material. Each day, at 0900 and $1700 \mathrm{~h}$, the cages were checked for the presence of newborn litters. The first day a newborn litter was present was recorded as Day 0. On Postnatal Day 1 the litters were culled to 8 pups; litters containing fewer than 8 pups were not used in these experiments. On the day of testing, the dams were removed and the pups were placed in a breeding cage with fresh litter. At this point, each pup was marked with a felt-tipped pen for later identification. The pups were then run singly in a separate room.

Experiments 1a, 1b, and 1c. The testing procedure for the first three experiments consisted of picking up each pup carefully by the nape of the neck with a spring-mechanized clothespin and suspending it approximately $44 \mathrm{~cm}$ above the surface of a table (see Figure 1). Some pilot work had alerted us to the fact that inconsistent pressure produced by swinging the animal might cause the subject to assume the TR. Because we were interested in producing, or potentiating, a TR with stimuli other than the dorsal pinch, we wanted to produce as weak a TR as possible with only the clothespin. We found that if we elevated the rat and clipped it with the clothespin very carefully it would relax and not show the classic TR as reported by Brewster and Leon (1980). At this point, each rat was given the first of an individually predetermined randomly ordered sequence of stimulus presentations. Immediately following a given presen-
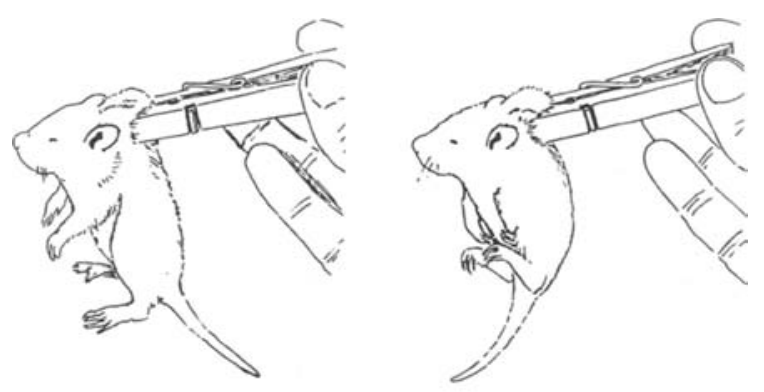

A

Figure 1. Drawing of 15-day-old rat pups being suspended with a spring-mechanized clothespin. (A) A pup wth no TR; (B) a pup with a TR score of 4 .

tation, the response was photographed for later scoring and the rat was placed back with its littermates. After all the pups in the litter had been given their first stimulus presentation, the procedure was repeated until each pup had received its entire sequence of stimulus presentations.

Experiments 2 and 3. Subjects in Experiments 2 and 3 were 33 and 19 days of age, respectively, at the time of testing and were too large to be held effectively with the clothespin. The procedures in Experiments 2 and 3 were, in general, the same as those described in previous research (Brewster \& Leon, 1980; Wilson, 1985; Wilson et al., 1984) The subjects were given a dorsal pinch by an experimenter's hand to elicit a TR. In addition, the subjects were given a series of independently determined randomly presented stimulus presentations. Again, each response was photographed for later scoring.

Scoring of the responses followed the schema proposed by Brewster and Leon (1980). Each pup's response was graded on a scale of $0-5$ with 1 point being awarded for each forelimb, hindlimb, and/or tail that the animal brought into contact with its ventrum after it had received the stimulus presentation.

\section{Data Analysis}

The data were analyzed using standard parametric procedures with subsequent comparisons between conditions being determined with Scheffé tests (Kirk, 1968). ${ }^{1}$ Differences with a probability $<.05$ $(p<.05)$ were considered statistically significant.

\section{EXPERIMENT 1a}

In this experiment, we sought to determine whether, when a pinch to the nape of the neck of a 15-day-old rat pup produced only a minimal TR, an additional tactile stimulus, in the form of an airpuff to the pup's ventrum, could induce or intensify the TR consistently. Subjects were given a trial with no stimulus presentation, a trial with an airpuff, and a trial with an auditory click.

\section{Procedure}

Thirty-two (four litters) 15-day-old rat pups were tested using a repeated measures design. For any given stimulus presentation, each pup was first suspended and then, within $2 \mathrm{sec}$, given one of a series of three independently ordered stimulus presentations. The treatments in this experiment were: (1) no additional stimulus, (2) an airpuff to the ventrum, and (3) an auditory click. This last stimulus was used because we were concerned that a general increase 
Table 1

Percentages of 15-Day-Old Pups Showing Specific TR

Intensities, and Intensity Means, as a Function of Experimental Condition (Experiment 1a)

\begin{tabular}{lccccccc} 
& \multicolumn{9}{c}{ Response Intensity } & Mean \\
\cline { 2 - 7 } Condition & 0 & 1 & 2 & 3 & 4 & 5 & TR \\
\hline No Stimulus & $94(30)$ & $3(1)$ & $3(1)$ & 0 & 0 & 0 & 0.09 \\
Airpuff & 0 & 0 & $56(18)$ & $34(11)$ & $9(3)$ & 0 & $2.53^{*}$ \\
Clicker & $81(26)$ & $3(1)$ & $16(5)$ & 0 & 0 & 0 & 0.34 \\
\hline
\end{tabular}

Note-Numbers in parentheses $=$ numbers of subjects showing a particular response intensity with $N=32$. *Reliably different from the no-stimulus control conditon.

in environmental stimulation and not the tactile stimulus itself actually might be a potentiating factor. The airpuff was a quick $(<1-$ sec) oral puff delivered by the experimenter and directed at the center of the subject's ventrum. The puff was strong enough to displace the hairs on the subject's ventrum but not strong enough to cause the subject's entire body to move. The click was delivered from a hand-held clicker positioned within $1 \mathrm{~cm}$ of the subject's right ear. We chose to deliver the airpuff orally. Pilot work for this study had shown that the TR could be induced with a light pinch supplemented with an airpuff from a hair dryer or a small bellows but that gently blowing on the pups produced the effect reliably with the fewest number of confounding variables (e.g., noise).

\section{Results and Discussion}

Mean response intensities and the percentage of subjects demonstrating specific response intensities for this experiment are presented in Table 1. A repeated measures analysis of variance (ANOVA) analyzing differences in mean response intensities revealed a reliable treatment effect $[F(2,62)=160.34]$. Post hoc analyses on these data revealed the TR to be more intense in the airpuff condition, with the auditory stimulus producing an effect not different from the no-stimulus situation. There was essentially no TR in the pups in the no-stimulus situation. On first consideration, one might be concerned with this result, but, in fact, the intent was to produce this exact effect-having the suspended pup be very relaxed and showing a minimal TR. The tactile stimulation produced by the airpuff was very effective in inducing the TR in rats while being suspended by the clothespin. The fact that the airpuff was able to induce a TR of 2 or higher in $100 \%$ of the rats seemed rather remarkable, since the clothespin itself induced a partial TR in only 3 of the 32 animals while under the no-stimulus control condition. The presentation of the auditory stimulus, in general, caused the pups to jerk immediately and then relax without showing a TR. This led us to the conclusion that tactile stimulation was necessary to produce the response.

\section{EXPERIMENT 1b}

In our second experiment, we sought to determine the generality of the effect reported in Experiment 1. To do this we used a brief tailpinch, instead of an airpuff, as the tactile stimulus. We compared responses under this condition with responses under a no-stimulus control condition and an auditory-stimulus condition.

\section{Procedure}

Twenty-four (three litters) 15-day-old rat pups were tested using a repeated measures design. The procedure was identical to that described in Experiment la except that the treatments in this experiment were (1) no additional stimulus, (2) a brief ( $<0.25-\mathrm{sec})$ tailpinch with a pair of mouse-toothed forceps, and (3) a $1000-\mathrm{Hz}$ tone at $60 \mathrm{~dB}$ for $0.5 \mathrm{sec}$ using a Hewlett-Packard Model 201C audio oscillator.

\section{Results and Discussion}

Data for this experiment are presented in Table 2. A repeated measures ANOVA on the mean response intensities revealed a reliable treatment effect $[F(2,46)=$ 113.76]. Scheffé tests revealed that the TR was significantly more intense in the pups given the tailpinch than in pups in either the no-stimulus control condition or the auditory-stimulus condition; the latter two groups did not differ from each other. Tactile stimulation, in this case in the form of the tailpinch, was again able to elicit the TR reliably. In addition, the tailpinch caused the rat pup to emit fairly consistently a brief squeak that was followed by an immediate, but very transient, struggle. Within $2 \mathrm{sec}$, the subjects assumed a fairly intense TR. What we

Table 2

Percentages of 15-Day-Old Pups Showng Particular TR

Intensities, and Intensity Means, as a Function of Experimental Condition (Experiment 1b)

\begin{tabular}{lccccccc}
\hline & \multicolumn{9}{c}{ Response Intensity } & Mean \\
\cline { 2 - 7 } Condition & 0 & 1 & 2 & 3 & 4 & 5 & TR \\
\hline No Stimulus & $79(19)$ & $17(4)$ & 0 & $4(1)$ & 0 & 0 & 0.29 \\
TallPinch & $4(1)$ & 0 & $13(3)$ & $42(10)$ & $37(9)$ & $4(1)$ & $3.21^{*}$ \\
Tone & $79(19)$ & $17(4)$ & $4(1)$ & 0 & 0 & 0 & 0.25 \\
\hline
\end{tabular}

Note-Numbers in parentheses $=$ numbers of subjects showing a particular response intensity with $N=24 . \quad *$ Reliably different from the no-stimulus control condition. 
observed was probably two different responses to two different aspects of the same stimulus presentation. The pup, initially perceiving the pinch, which may have been uncomfortable, may have attempted actively to avoid the pinch by struggling. Once the actual stimulus was no longer present, the pup assumed a TR. Lending credence to this conclusion is the observation that the airpuff presented in Experiment la did not cause any of the pups to squeak or to struggle. As before, auditory stimulation was ineffective in producing any TR change in the control condition.

\section{EXPERIMENT 1c}

At this point, we chose to investigate whether a sudden visual stimulus would produce a change in the intensity of the TR of animals suspended from a clothespin. We compared responses under this condition with TRs under a no-stimulus control condition and under a tactilestimulation condition.

\section{Procedure}

Sixteen (two litters) 15-day-old rat pups were tested using a repeated measures design. Each pup was suspended as described above, with treatments in this experiment being (1) no additional stimulus, (2) a flash of light from a camera flash attachment, and (3) an airpuff as in Experiment 1a. We chose the last stimulus condition as a control to make certain that the animals were capable of assuming the TR. The flash attachment, a Philips P36 Computer TLS unit, was held $2 \mathrm{~cm}$ from the suspended rat's face and flashed. The procedure for presenting the airpuff was identical to that described for Experiment la.

\section{Results and Discussion}

Data for this experiment are presented in Table 3. An ANOVA on mean response intensities revealed a significant treatment effect $[F(2,30)=85.16]$. Post hoc analyses revealed that the airpuff was able to induce a relatively intense TR in the pups and that there was no difference between the TR intensity following the light flash and the TR intensity in the no-stimulus condition. In the light-flash situation, the pups showed no change in behavior at all. The pups in this experiment were tested in a fairly well-lit room. We considered the lack of effect with the light flash as perhaps being due to too little difference between the relative amounts of ambient light and stimulus light. We subsequently took a litter of animals, tested them under conditions of very low ambient light, and found that the light flash still had no noticeable effect on their behavior.

In summary, results from Experiments $1 \mathrm{a}, 1 \mathrm{~b}$, and $1 \mathrm{c}$ indicate that, when a pinch to the dorsal surface of a rat pup is ineffective in producing a TR, additional tactile stimulation to an area that has previously been shown to be ineffective in producing the response can induce a TR.

\section{EXPERIMENT 2}

Brewster and Leon (1980) reported that elicitation of the TR was a transient phenomenon. According to them, because the presence of the response is dependent upon a response hierarchy, competing responses such as defensive behaviors will suppress the TR by the 4th postpartum week. This change in response hierarchy might be a function of endogenous neurotransmitter systems' suppressing the neurochemical system(s) underlying the TR. Reasoning that the neurochemical mechanisms that subserve the TR are suppressed in older animals, we chose to investigate the ability of additional tactile stimulation to reinstate a TR in 33-day-old rats. We hypothesized that if the TR elicited by the supplemental tactile stimulation was a function of a neurochemical system other than the dopaminergic system, we would be able to elicit a TR with additional stimulation even if a strong pinch to the nape of the neck proved to be an inadequate stimulus.

\section{Procedure}

Thirty-two (four litters) 33-day-old rats were tested using a repeated measures design. Each rat was suspended by the nape of the neck with an experimenter's thumb and first two fingers. At this point, the rat was given two test trials: (1) no additional stimulus or (2) a puff of distilled water from an atomizer held $1 \mathrm{~cm}$ from the rat's face. The order of stimulus presentations was random and individually predetermined for each rat. Some pilot work had shown that there was no difference in the TR intensities within animals when they were given either the mist of water or an airpuff. We chose to use the mist from an atomizer, though, because we thought that it might provide more consistent stimulation between animals.

\section{Results and Discussion}

Data for Experiment 2 are presented in Table 4. A $t$ test on response intensities revealed a significant treatment effect $\left[t_{d}(31)=6.96\right]$. It appears that we can reinstate a fairly intense TR in rats that are no longer capable of showing a TR under normal conditions. There are two

Table 3

Percentages of 15-Day-Old Pups Showing Particular TR Intensities, and Intensity Means, as a Function of Experimental Condition (Experiment 1c)

\begin{tabular}{|c|c|c|c|c|c|c|c|}
\hline \multirow[b]{2}{*}{ Condition } & \multicolumn{6}{|c|}{ Response Intensity } & \multirow{2}{*}{$\begin{array}{c}\text { Mean } \\
\text { TR }\end{array}$} \\
\hline & 0 & 1 & 2 & 3 & 4 & 5 & \\
\hline No Stimulus & $81(13)$ & $13(2)$ & $6(1)$ & 0 & 0 & 0 & 0.25 \\
\hline Airpuff & 0 & 0 & $19(3)$ & $44(7)$ & $37(6)$ & 0 & $3.19^{*}$ \\
\hline Light Flash & $88(14)$ & $\mathbf{0}$ & $6(1)$ & $6(1)$ & 0 & 0 & 0.31 \\
\hline
\end{tabular}

Note-Numbers in parentheses $=$ numbers of subjects showing a particular response intensity with $N=16$. *Reliably different from the no-stimulus control condition. 
Table 4

Percentages of 33-Day-Old Pups Showing Particular TR

Intensities, and Intensity Means, as a Function of Experimental Condition (Experiment 2)

\begin{tabular}{lccccccc}
\hline & \multicolumn{9}{c}{ Response Intensity } & Mean \\
\cline { 2 - 7 } Condition & 0 & 1 & 2 & 3 & 4 & 5 & TR \\
\hline No Stimulus & $66(21)$ & $22(7)$ & $9(3)$ & $3(1)$ & 0 & 0 & 0.50 \\
Water Puff & $19(6)$ & $22(7)$ & $9(3)$ & $38(12)$ & $13(4)$ & 0 & $2.03^{*}$ \\
\hline
\end{tabular}

Note-Numbers in parentheses $=$ numbers of subjects showing a particular response intensity

with $N=32$. *Reliably different from the no-stimulus control condition.

possible explanations for these results. The first is that reinstatement of the TR was caused by a system other than a dopaminergic system. A perhaps more parsimonious explanation is that the additional stimulation caused a dopamine system to overcome its endogenous suppression, which resulted in a more intense TR.

\section{EXPERIMENT 3}

This experiment was designed to further investigate whether the increment in TR intensity following supplemental tactile stimulation was subserved by a dopaminergic system or if the increase in TR intensity was due to a compensatory neurochemical system. This experiment was designed to replicate previous work (Wilson et al., 1984) by testing for TR intensity in 19-dayold rat pups after the pups had been given varying doses of haloperidol, a potent dopaminergic receptor blocker (Siegel et al., 1981). During half the trials, the rat pups were given supplemental airpuffs, as described in Experiment 1a, while they were being grasped by the nape of the neck. It was hypothesized that if the increment in response intensity produced with the ventral airpuff was due to dopamine release, since the postsynaptic receptors would be blocked, there would be no differences in response intensities in the pups given haloperidol, regardless of whether or not they were given airpuffs.

\section{Procedure}

Forty (five litters) 19-day-old rat pups were tested using a repeated measures design. The pups were divided into three groups: a control group $(n=14)$, which was given a subcutaneous injection of isotonic saline $(5 \mathrm{ml} / \mathrm{kg})$, one experimental group $(n=13)$ that was given a subcutaneous injection of $2.5 \mathrm{mg} / \mathrm{kg}$ haloperidol, and a second experimental group $(n=13)$, which was given a subcutaneous injection of $5.0 \mathrm{mg} / \mathrm{kg}$ haloperidol. Following a 20 -min postadministration delay, the pups were given a series of four randomly presented and independently determined test trials for TR intensity. Two trials consisted of pinching the pups by the nape of the neck as described in Experiment 2, and two trials consisted of the pinch combined with the delivery of airpuffs as described in Experiment la. The airpuff was used again because of its ease of delivery and because pilot work had shown that there were no differences between TR under an airpuff condition and TR under the atomizer condition. Since we used more than one trial for each stimulus presentation, data consisted of mean response intensities for each of the groups.

\section{Results and Discussion}

Data for this experiment are presented in Table 5. An ANOVA on mean response intensities revealed a reliable dose effect $[F(2,37)=24.19]$, a reliable trial effect $[F(1,37)=108.71]$, and reliable dose $\times$ treatment interaction $[F(2,37)=21.40]$. Post hoc analyses revealed a reduction in TR intensity with haloperidol, regardless of dose, relative to the saline-control rats. The airpuff reinstated the TR intensity to levels not different from those of the control subjects in the pups that received haloperidol. These results are in agreement with previous findings (Wilson et al., 1984) that pups' response to a pinch on the nape of the neck is subserved by a dopaminergic system. The results also point to the conclusion that the response to the airpuff is perhaps a compensatory response, not necessarily subserved by dopamine. During the course of this experiment, we noticed, in the slides used for scoring, that in the pups that had received an airpuff after having received haloperidol, the form of the response seemed to change. Rather than adducting their hindlimbs straight up to the ventrum, as did the control animals, the pups given haloperidol adducted their hindlimbs up and toward the medial plane, giving them the appearance of being pigeon-toed. We took this reduction in fine motor control as evidence of a loss of dopaminergic activity. The fact that these pups still

Table 5

Mean TR Intensities of 19-Day-Old Pups as a Function of Experimental Condition (Experiment 3)

\begin{tabular}{|c|c|c|c|c|}
\hline \multirow[b]{2}{*}{ Condition } & \multicolumn{2}{|c|}{ Hand Pinch } & \multicolumn{2}{|c|}{ Hand Pinch + Airpuff } \\
\hline & Mean & $S E M$ & Mean & $S E M$ \\
\hline Saline & 3.04 & .20 & 3.21 & .09 \\
\hline Haloperidol $(2.5 \mathrm{mg} / \mathrm{kg})$ & 1.00 & .20 & 2.92 & .17 \\
\hline Haloperidol $(5.0 \mathrm{mg} / \mathrm{kg})$ & 0.88 & $.19^{*}$ & 3.00 & .23 \\
\hline
\end{tabular}

*Reliably different from the control condition. 
showed a TR we took as evidence of a compensatory mechanism being brought into play with the supplemental stimulation. Interestingly, in reviewing the slides of the rats from Experiment 2, we noted that some of these older animals also showed this medial-plane response.

\section{GENERAL DISCUSSION}

Tactile stimulation is very important to the young, nonprecocial animal. Data presented in Experiments 1a, lb, and $1 \mathrm{c}$ indicate that if a pinch by an experimenter does not induce a strong TR, additional tactile stimulation can induce the response or intensify a weak TR. The TRs reported here were a function of tactile stimulation combined with the pinch on the nape of the neck. Pilot work in our lab had shown that freely moving pups given an airpuff or pups held by their tails in an inverted position and given an airpuff do not show the TR. Thus, in these circumstances, both the pinch and the additional tactile stimulation are necessary; neither alone is sufficient.

Under the assumption that the experimenter's pinch simulates the mother's grasp during transport, the results reported here indicate that if the mother does not have a firm grip on the pup or if the pup's sensory mechanisms specific to the bite on the nape of the neck are inadequate to convey successfully information about the mother's grasp, additional tactile stimulation can provide enough supplemental stimulation to induce the TR.

In 33-day-old rats, the TR is weakened considerably, indicating that the systems subserving the TR are perhaps suppressed. A puff of water from an atomizer to the face, though, can elicit the response fairly reliably in these animals. In 19-day-old rats, suppressing the proposed mechanism pharmacologically with haloperidol likewise inhibits the TR; again, the response can be elicited with additional tactile stimulation. These results indicate that perhaps the potentiation of the TR with supplemental tactile stimulation may be mediated by a neurochemical system other than dopamine; even in pups that had their dopamine systems blocked with very high levels of haloperidol, we were able to elicit the response with a quick airpuff.
Perhaps the phenomenon of response potentiation by supplemental tactile stimulation is a compensatory mechanism that is brought into play when the primary mechanisms are not fully developed or fully functional. Work in our laboratory is currently aimed at providing a more definitive explanation of the mechanisms underlying the TR response potentiation reported here.

\section{REFERENCES}

Brewster, J., \& LeON, M. (1980). Facilitation of maternal transport by Norway rat pups. Journal of Comparative \& Physiological Psychology, 94, 80-88.

KIRK, R. E. (1968). Experimental design: Procedures for the behavioral sciences. Belmont, CA: Brooks/Cole.

KLeiman, D. G. (1972). Maternal behavior of the green agouchi (Myoprocta pratti pocock), a South American caviomorph rodent. Behaviour, 43, 48-84.

Lorzou, L. A. (1969). The development of monoamine-containing neurones in the brain of the albino rat. Journal of Anatomy, 104, 588.

Lozzou, L. A. (1972). The postnatal ontogeny of monoamine-containing neurons in the central nervous system of the albino rat. Brain Research, 40, 395-418.

SAUER, E. G. F. (1967). Mother-infant relationship in galagos and the oral child-transport among primates. Folia Primatologia, 7, 127-149.

Siegel, G. J., Albers, R. W., Agranoff, B. W., \& Katzman, R. (1981). Basic neurochemistry (3rd ed.). Boston: Little, Brown.

SPEAR, L. P., \& Ristine, L. A. (1982). Suckling behavior in neonatal rats: Psychopharmacological investigations. Journal of Comparative \& Physiological Psychology, 96, 244-245.

WILSON, C. (1985). The effects of apomorphine and isoproterenol on the "transport response" in the white rat. International Journal of Developmental Neuroscience, 3, 279-284.

Wilson, C., Cullen, E., Sendell, K. (1984). A pharmacologic investigation of the "transport response" in the white rat. International Journal of Developmental Neuroscience, 2, 323-329.

\section{NOTE}

1. Appropriate nonparametric statistical techniques were applied to the data from each experiment as a check for the parametric statistical tests reported in the results sections. The nonparametric test results were not reported, as they were identical to the parametric statistical test results.

(Manuscript received May 28, 1987; revision accepted for publication August 6, 1987.) 